\title{
Determination of Electrical and Optical Design Parameters of High-Efficiency OLED
}

\author{
Ipek Atik ${ }^{1}$, O. Faruk Farsakoglu ${ }^{2}$, Nurdal Watsuji ${ }^{3}$, Saban Yilmaz ${ }^{4}$ \\ Kilis 7 Aralik University, Faculty of Engineering Architecture, Department of Electrical Electronics Engineering, \\ Kilis, 079000, Turkey ${ }^{1,2,4}$
}

Gaziantep University, Faculty of Engineering, Department of Electrical and Electronics Engineering, Gaziantep, 27000, Turkey ${ }^{3}$

\begin{abstract}
Although LED (Light Emitting Diode) was discovered in the 1900s, in recent years, it is utilized in lighting, screens and many indoor and outdoor applications widely. On the other hand, OLED (Organic Light Emitting Diode) technology is one of the advancing branches of LED technology. OLEDs, differently from other ordinary LEDs, contain organic light emitting layers between two electrical contacts. OLEDs are very slim and flexible. They are capable of providing more vivid and brighter display compared to LCDs (Light Crystal Displays). In our study, information about structural characteristics of OLED has been given. Band model of OLED has been explained, and functional equalities have been included. A three layer OLED has been designed. Optic materials with high light efficiency such as IRPPY, BCP, and Alq3 have been used in the structure of OLED. In the analyses, General-purpose Photovoltaic Device Model - gpvdm simulation software has been used. Bandwidth values of the layers have been determined respectively as $3 \mathrm{e}^{-08}, 5 \mathrm{e}^{-08}$ and $2 \mathrm{e}^{-08}$. As optical materials, Indium Tin Oxide (ITO) for anode and Silver (Ag) for cathode have been used. To the OLED designed, in an electrical circuit with current limiting, a voltage between $-0.2 \mathrm{~V}$ and $2.5 \mathrm{~V}$ has been implemented, and light output power variance curves have been obtained. On OLED band structure, energy level variance curves of lowest unoccupied molecular orbital (LUMO) and highest occupied molecular orbital (HOMO) have been obtained according to Maxwell-Boltzmann statistical features. Photon density emitted by optical materials used, and energy and location variance data have been obtained. Efficiency values of designed OLED have been aimed to be at optimum level. In order for HOMO and LUMO energy levels to stay at equilibrium state, bandwidth values have been analyzed. The study has been aimed to be a pathfinder in the direction of being able to analyze effects of OLED technology and organic layers on energy level.
\end{abstract}

Key Words: OLED, band model, HOMO - LUMO energy, optic materials.

\section{INTRODUCTION}

\section{A. OLED (Organic Light Emitting Diode)}

OLED (Organic Light Emitting Diode) is an advanced version of LED (Light Emitting Diode) which has been developed as an alternative to LCD (Light Crystal Display, Liquid Crystal Display) technology. What makes OLEDs differ from other LEDs is that there is a thin-film organic layer which provides the radiance in it. Structurally, it consists of a series of thin organic film layers between electrical contacts. OLEDs consist of organic materials with low molecular weigth or polymer-based materials. The thickness of OLEDs is $100-500 \mathrm{~nm}$. In their structures, there are two or three organic layers. In order for them to be able to work as semiconductors, organic light emitting material should have a connection with conjugate $\pi$. First time in 1965, OLEDs were spotted due to aromatic anthracene material emitting blue light as a result of being put in between two electrodes. Thereafter, in the late 1980s, Tang, VanSlyke and Saito Tsutsui developed different versions of OLEDs by using different organic fluorescent painting materials [1-2]. In the 1990s, Friend $\mathrm{Vr}$ Group have used polymer poly (p-phenylene vinylene_PPV) which is an active material in single layer OLED and demonstrates better fluorescence features.
Afterwards, PPV has been started to be used in OLED technology. Therefore, the number of molecules having luminance in a simple way to the number of total activated molecule (quantum efficiency) ratio has shown a $100 \%$ increase [3]. Chemicals that do not have fluorescence features have a near-zero quantum efficiency rate. Many materials have been analyzed in the direction of increasing OLED performance. According to this, materials have been distributed into four groups; conjugated polymers, low molecular weight materials, conjugated oligomers and chromophores insulated to main and side polymer chains [4].

For the white light emitting OLEDs, donor-acceptor structure is usually used. On the structures for which donor-acceptor structure is used, high energy emitting donor material is doped with low energy acceptor material; and energy transfer is realized in this way [5]. Materials used in donor-acceptor system emit wider in the solid state compared to them being in the solvent. The reason for this is intermolecular interaction in solid materials being more. This leads to a better emission and increases quality. 


\section{B. OLED Structure}

The OLED structure consists of a few layers. These are cathode, electron transport layer, layer where organic materials are located, hole transport layer, indium tin oxide anode and glass layer. OLED structure layers are shown in Fig.1.

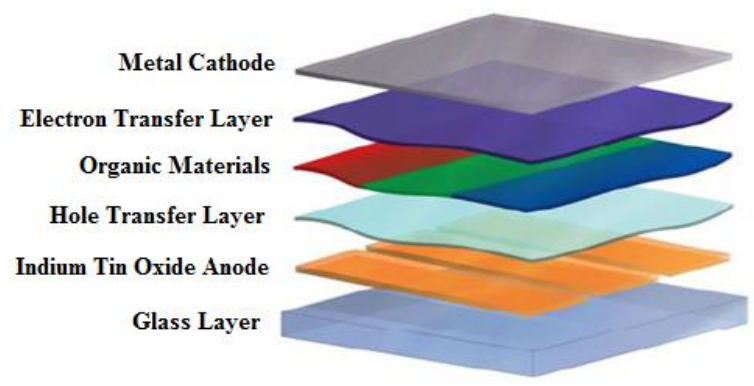

Fig.1. OLED layers

The material used for the anode is usually indium tin oxide (ITO). For the cathode, $\mathrm{Ca}, \mathrm{Mg}, \mathrm{Al}$ or low function materials, which are alloys of those, are used. Organic layer in between should be able to transport electrons well. In cases where the voltage is given to OLED device, $h^{+} \mathrm{s}$ are transported from HOMO level in radical cation form, and $\mathrm{e}^{-} \mathrm{s}$ are transported from LUMO level in radical anion form. Anions and cations moving by the effect of electric field meet at any location of emission layer; $\mathrm{e}^{-} \mathrm{s}$ and $\mathrm{h}^{+} \mathrm{s}$ are combined (electron-hole recombination) and constitute a light emission. Electron and hole encounters being at an equal rate is an important point for OLED to run [6]. The energy level diagram of two-layer OLED device has been given in Fig.2.

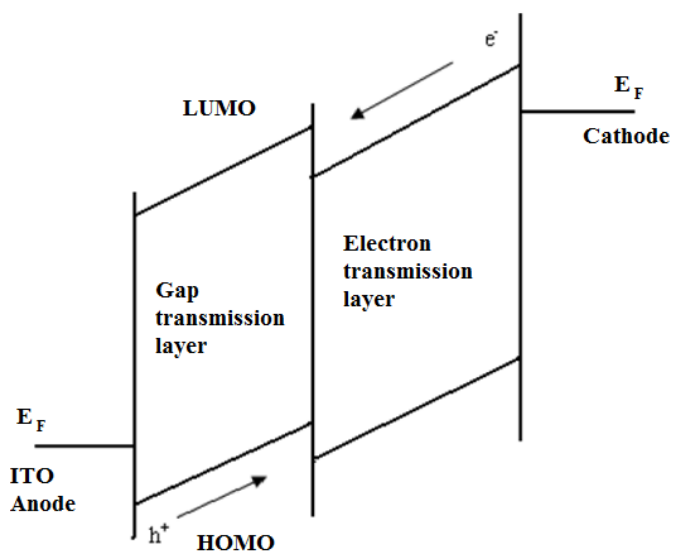

Fig.2. Energy level diagram of two-layer OLED.

C. Advantages and Disadvantages of OLEDs

Organic structured LEDs have many advantages compared to other organic LEDS. OLEDs can be used as around $100-500 \mathrm{~nm}$ very thin-film. Because they are thin and flexible, they are foldable. For this reason, it is more resistant to mechanical impacts. They run under low voltage; for this reason, they have low energy consumption. Thanks to the organic materials, color tone, which will be given emission, can be adjusted [41]. With OLEDs, it is possible to have a more vivid image acquisition containing a higher brightness value $\left(15 \cdot 10^{4}\right.$ $\mathrm{cd} / \mathrm{m}^{2}$ ) compared to LCDs. Response time is much shorter compared to other LCDs. While response time of LCD screens are varying between 2 and $8 \mathrm{~ms}$, with OLEDs, this time is much lesser than 0.01. OLED screens offer a much wider visual angle (approximately $170^{\circ}$ ) than other LCD screens. On the other hand, disadvantages of OLEDs are sensitiveness to humidity and oxygen. Accordingly, device lifetimes are low.

\section{OLED Band Structure}

Bandwidth with OLEDs is the energy difference between lowest unoccupied molecular orbital (LUMO) and highest occupied molecular orbital (HOMO) for functional $\pi$ conjugated systems. It is among the factors affecting the system performance in the production of devices such as transistors, light-emitting diodes, field-effect transistors and solar batteries.

Bandwidth energy may vary according to several factors. Adjusting HOMO-LUMO energy levels affects system performance in a remarkable way [7]. In Fig.3, factors affecting bandwidth are shown in molecular structure.

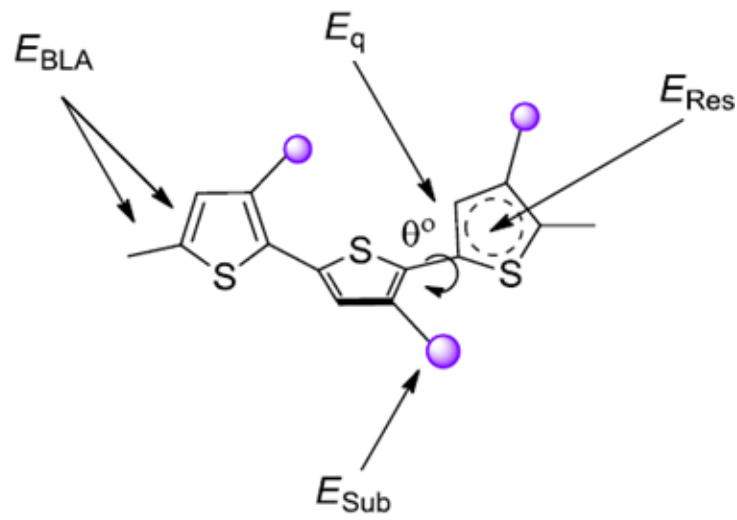

Fig.3. Factors affecting bandwidth shown in molecular structure

Factors affecting bandwidth as shown in the figure are planarity, resonance energy, intermolecular interaction, bond length variance and substituent factors. Effect of these expressions on device performance has been given as functional equation equivalence;

$$
\Delta E=E_{g}=E_{B L A}+E_{\mathrm{Res}}+E_{\theta}+E_{S u b}+E_{\text {Int }}
$$

As it is seen in Figure 3, $\mathrm{E}_{\mathrm{g}}$; stands for total energy, $\mathrm{E}_{\mathrm{BLA}}$; for energy variances based on bond length variances, $\mathrm{E}_{\mathrm{RES}}$; for resonance energy, $\mathrm{E}_{\mathrm{sub}}$; for substituent energy, $\mathrm{E}_{\theta}$; for energy variances based on $\theta$ angle, $\mathrm{E}_{\text {int }}$; for internal quantum energy.

\section{DESİGN AND ANALYSIS}

An OLED system performance varies based on bandwidth and features of material used in the system. In this section, electrical and optical design parameters of OLED have been determined. In the direction of determined parameters, optical and electrical features have been analyzed in order to evaluate OLED system efficiency. 
System performance evaluations have been performed. In the analyses, Photovoltaic Device Design software Version 2016 has been used. Diagrammatic representation belonging to designed OLED is given in Fig. 4. Materials used for the design and bandwidth values data of materials are given in Table 1.

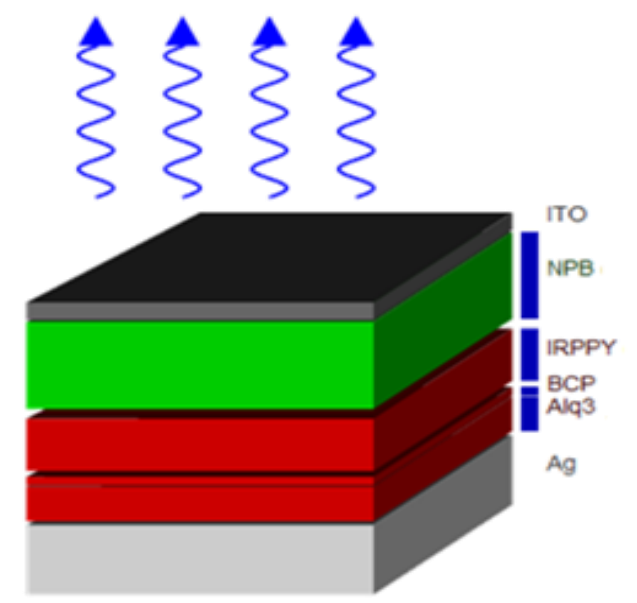

Fig.4. OLED diagrammatic representation

Optic materials with high light efficiency such as IRPPY, $\mathrm{BCP}$, and Alq3 have been used in the structure of OLED. Bandwidth values of the layers have been determined respectively as 3.e-08, 5.e-08 and 2.e-08. As optical materials, Indium Tin Oxide (ITO) for anode and Silver (Ag) for cathode have been used.

Table 1. Layers of OLED, optical material used for layers and bandwidth values

\begin{tabular}{|l|l|c|}
\hline $\begin{array}{l}\text { Layer } \\
\text { Name }\end{array}$ & \multicolumn{1}{|c|}{$\begin{array}{c}\text { Optical } \\
\text { Material }\end{array}$} & $\begin{array}{c}\text { Band } \\
\text { Gap (m) }\end{array}$ \\
\hline ITO (anot) & ITO & $1 \mathrm{e}^{-08}$ \\
\hline NPB & NPB & $5 \mathrm{e}^{-08}$ \\
\hline IRPPY & Generic_Organic & $3 \mathrm{e}^{-08}$ \\
\hline BCP & Generic_Organic & $5 \mathrm{e}^{-08}$ \\
\hline Alq3 & Generic_Organic & $2 \mathrm{e}^{-08}$ \\
\hline Ag (katot) & Ag & $4 \mathrm{e}^{-08}$ \\
\hline
\end{tabular}

Ambient temperature during analyses is determined as 300 ${ }^{\circ} \mathrm{K}$. As circuit diagram, a simple current limiting OLED circuit with ballast resistor has been used, and circuit diagram has been given in Figure 5. The series resistance value is $1 \Omega$, shunt resistance value is $1 \mathrm{e}^{10} \Omega^{\prime}$ and capacitance value is $1 \mathrm{e}^{26}$ in the circuit.

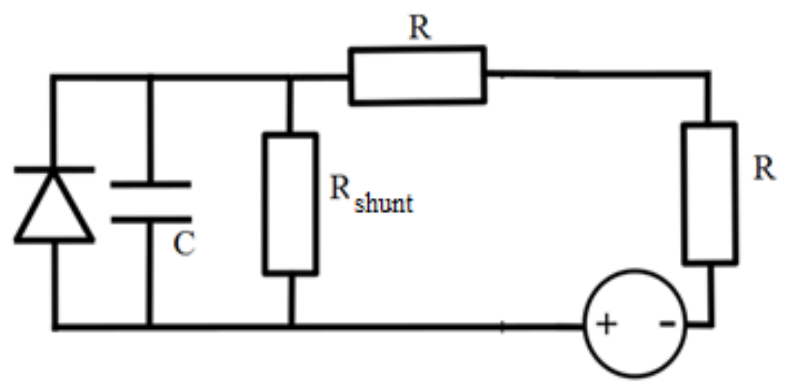

Fig.5. Electrical circuit diagram of OLED
Voltage implemented to OLED differed between $-0.2 \mathrm{~V}$ and $2.5 \mathrm{~V}$. Maximum current density is $1500.0 \mathrm{~A} \mathrm{~m}^{-2}$. Current-voltage variance, voltage-light output power variance, and location-total current density variances occurred in OLED based on these parameters are given respectively in Figure 6 and Figure 7.

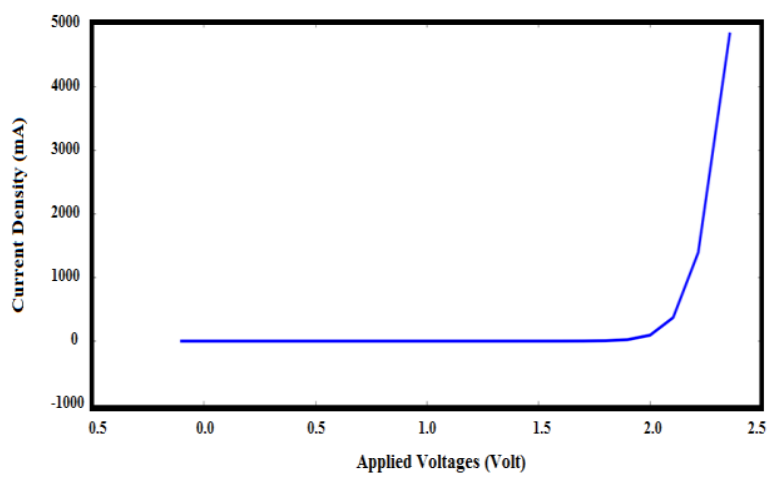

Fig.6. Current-voltage variance of OLED

Current limiting has been implemented for OLED by using series and shunt resistances. On the electric circuit, voltage values between $-0.2 \mathrm{~V}$ and $2.5 \mathrm{~V}$ have been implemented. While current and light output values of the circuit have stayed fixed until threshold voltage value of OLED exceeded $2 \mathrm{~V}$, then they have shown a linear increase. Maximum current density has exceeded the value of approximately $5 \mathrm{~A}$.

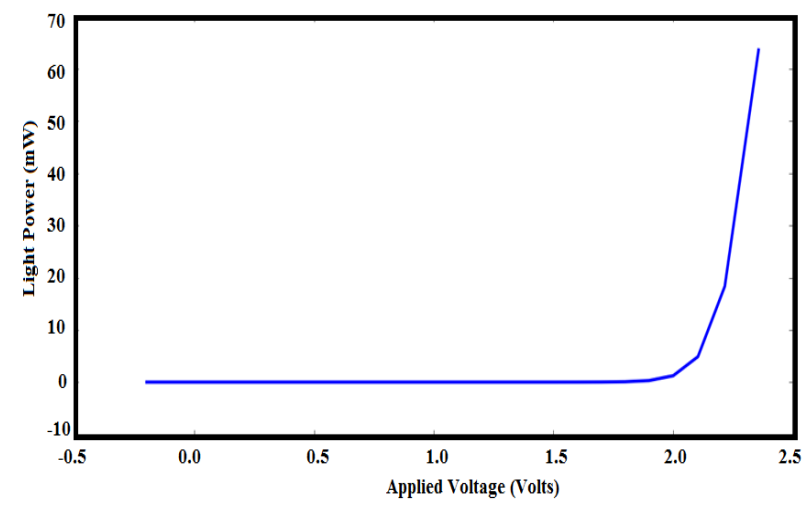

Fig.7. Voltage-light power variance implemented on OLED

Current limiting has been implemented for OLED by using series and shunt resistances. On the electric circuit, voltage values between $-0.2 \mathrm{~V}$ and $2.5 \mathrm{~V}$ have been implemented. While current and light output power values of the circuit have stayed fixed until threshold voltage value of OLED exceeded $2 \mathrm{~V}$, then they have shown a linear increase. Maximum light power has exceeded the value of $60 \mathrm{~mW}$.

In order to be able to calculate the electrical potential of OLED, transporter concentrations in $\mathrm{n}$ and $\mathrm{p}$ contacts in the structure of OLED, effective densities of $\mathrm{N}_{\text {Lumo }}$ ve $\mathrm{N}_{\text {номо }}$ states and effective bandwidth values should be known. OLED band structure in the equilibrium state is given in Figure 8. 


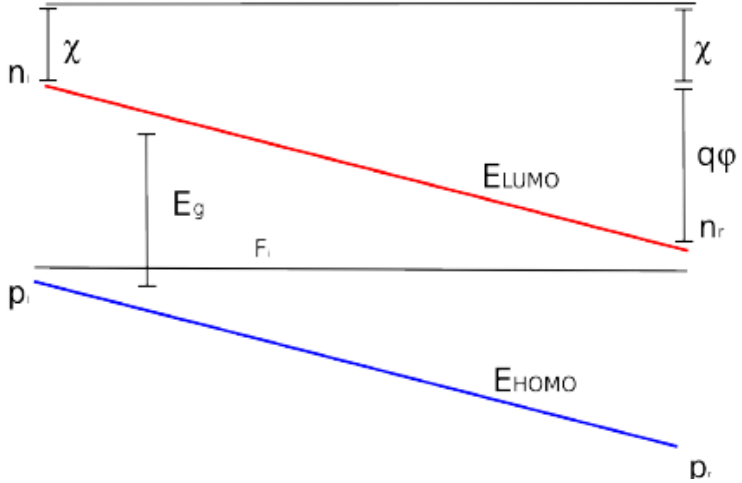

Fig.8. Band structure for OLED in the equilibrium state

When energy expression of $0 \mathrm{~V}$ is taken as reference here for LUMO and HOMO;

$$
\begin{aligned}
& E_{\text {LUМо }}=-x \\
& E_{\text {Номо }}=-x-E g
\end{aligned}
$$

Here Maxwell-Boltzmann statistics method can be used to be able to calculate Fermi Level $\left(\mathrm{F}_{\mathrm{i}}\right)$. Accordingly;

$$
\begin{aligned}
& p \imath=N_{v} \exp \left(\frac{E_{\text {Номо }}-F_{p}}{k T}\right) \\
& n \iota=N_{c} \exp \left(\frac{F_{n}-E_{L U M O}}{k T}\right)
\end{aligned}
$$

In the case of an equilibrium state, Fermi level has to be constant. In addition, there is a potential formed between the valence band and transmission band. Accordingly;

$$
\begin{gathered}
E_{v}=-x-q \phi \\
E_{v}=-x-E g-q \phi \\
n r=N_{c} \exp \left(\frac{F_{n}-E_{\text {LUMO }}}{k T}\right) \\
p r=N_{v} \exp \left(\frac{E_{v}-F_{\text {HOMO }}}{k T}\right)
\end{gathered}
$$

Energy location evaluation of analyzed OLED has been performed according to values of materials used and bandwidth. Data regarding LUMO, HOMO and Fi energy level are shown in Fig.9.

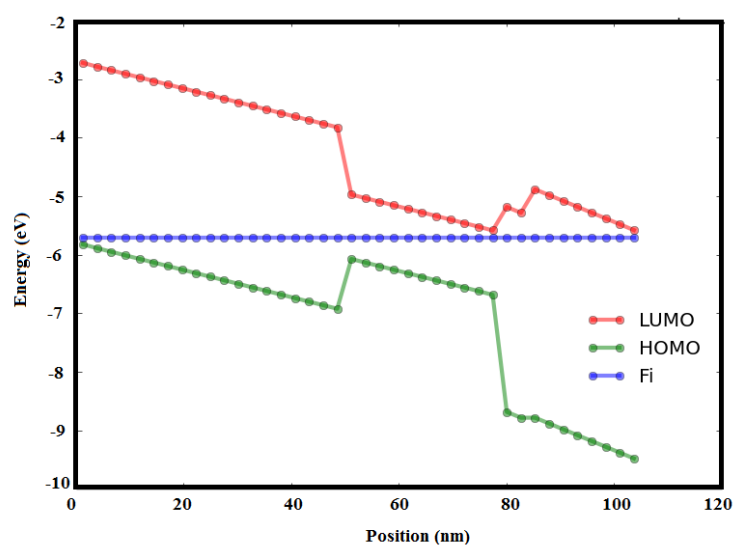

Fig. 9. HUMO-LUMO energy level variance of analyzed OLED
Photon density and energy variance data of optical material used for OLED structure have been given as location-dependent. It has been observed that Fermi energy level had remained constant in $0-100 \mathrm{~nm}$ position gap of OLED and been at approximately $-6 \mathrm{eV}$ value. However, it has been seen that increase and decrease in the HOMO - LUMO energies had been balanced. Maximum energy has been obtained as $2.5 \mathrm{eV}$ value at approximately $50 \mathrm{~nm}$ position of OLED. As it is seen in Figure 9, proportionally with energy decrease of LUMO level, HOMO energy level has increased. Photon density energy variance of OLED layers is given in Fig. 10.

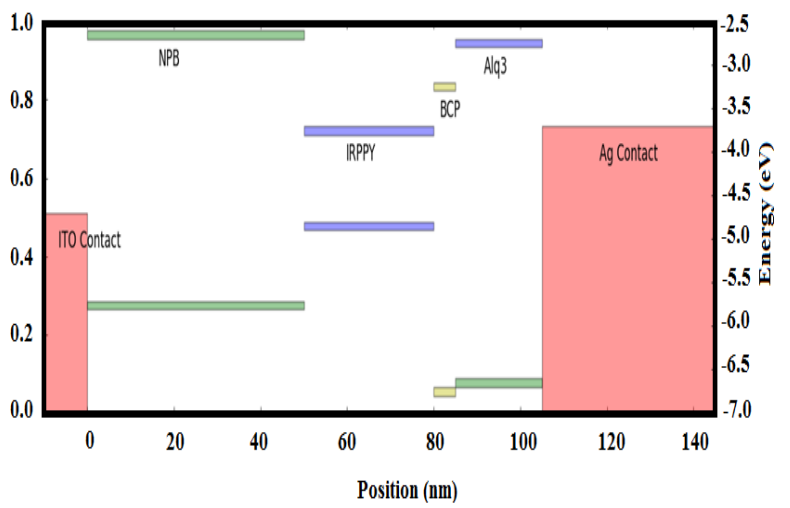

Fig.10. Photon density-location- energy variance of analyzed OLED

As it is seen in Fig. 10, photon densities and energy variances on location-dependent layers can vary based on parameters of analyzed OLED. Optic materials with high light efficiency such as IRPPY, BCP, and Alq3 have been used in the structure of OLED. In the analyses, Energy values of the layers have been determined. As optical materials, Indium Tin Oxide (ITO) for anode and Silver (Ag) for cathode have been used.

\section{CONCLUSIONS}

In our study, information about structural characteristics of OLED has been given. Band model of OLED has been explained, and functional equalities have been included. A three layer OLED has been designed. Optic materials with high light efficiency such as IRPPY, BCP, and Alq3 have been used in the structure of OLED. Bandwidth values of the layers have been determined respectively as $3 \mathrm{e}^{-08}, 5 \mathrm{e}^{-}$ 08 , and $2 \mathrm{e}^{-08}$. The OLED design has been performed according to Maxwell-Boltzmann statistics based upon characteristic features of OLED structure. Current limiting has been implemented for OLED by using series and shunt resistances. On the electric circuit, voltage values between $-0.2 \mathrm{~V}$ and $2.5 \mathrm{~V}$ have been implemented. While current and light output values of the circuit have stayed fixed until threshold voltage value of OLED exceeded $2 \mathrm{~V}$, then they have shown a linear increase. Maximum light power has exceeded the value of $60 \mathrm{~mW}$. Photon density and energy variance data of optical material used for OLED structure have been given as location-dependent. It has been observed that Fermi energy level had remained constant in $0-100 \mathrm{~nm}$ position gap of OLED and been at 
approximately $-6 \mathrm{eV}$ value. However, it has been seen that increase and decrease in the HOMO - LUMO energies had been balanced. Maximum energy has been obtained as $2.5 \mathrm{eV}$ value at approximately $50 \mathrm{~nm}$ position of OLED. Results obtained are applicable for advancing OLED technology.

\section{REFERENCES}

[1] C. Adachi, T. Tsutsui, and S. Saito, "Blue Light-Emitting Organic Electroluminescent Devices," Applied Physics Letters, vol. 56, pp. 799-801, Feb 261990.

[2] C. W. Tang and S. A. Vanslyke, "Organic Electroluminescent Diodes," Applied Physics Letters, vol. 51, pp. 913-915, Sep 21 1987.

[3] M. A. Baldo, D. F. O'Brien, Y. You, A. Shoustikov, S. Sibley, M. E. Thompson, et al., "Highly efficient phosphorescent emission from organic electroluminescent devices," Nature, vol. 395, pp. 151-154, Sep 101998.

[4] U. Mitschke and P. Bauerle, "The electroluminescence of organic materials," Journal of Materials Chemistry, vol. 10, pp. 1471-1507, 2000.

[5] A. Kumar, R. Srivastava, S. S. Bawa, D. Singh, K. Singh, G. Chauhan, et al., "White organic light emitting diodes based on DCM dye sandwiched in 2-methyl-8-hydroxyquinolinolatolithium," Journal of Luminescence, vol. 130, pp. 1516-1520, Aug 2010.

[6] Shinar, J., 2004, Organic Light-emitting Devices, Springer, New York, USA.

[7] J. Roncali, "Molecular engineering of the band gap of pi-conjugated systems: Facing technological applications," Macromolecular Rapid Communications, vol. 28, pp. 1761-1775, Sep 32007. 